IOS Press

\title{
Plasma 3,3',5-Triiodo-L-thyronine [T3] level mirrors changes in tumor markers in two cases of metastatic cancer of the breast and pancreas treated with exogenous L-T3
}

\author{
Alejandro Rodríguez-Molinero ${ }^{\mathrm{a}, *}$, Aleck Hercbergs ${ }^{\mathrm{b}}$, Manuel Sarrias ${ }^{\mathrm{c}}$ and Antonio Yuste ${ }^{\mathrm{a}}$ \\ ${ }^{a}$ Clinical Research Unit, Consorci Sanitari del Garraf, Vilanova i la Geltrú, Spain \\ ${ }^{\mathrm{b}}$ Departments of Radiation Oncology and Neuroradiology, Cleveland Clinic, Cleveland, OH, USA \\ ${ }^{\mathrm{c}}$ Department of Radiology, ConsorciSanitari del Garraf, Vilanova i la Geltrú, Spain
}

\begin{abstract}
Preclinical studies have attributed 3,3',5-triiodo-L-thyronine (T3) a direct negative effect on tumor progression, as well as chemosensitizing, differentiating and immunomodulatory properties. On the other hand, L-thyroxine (T4), via a thyroid hormone receptor on plasma membrane integrin $\alpha \mathrm{v} \beta 3$, promotes solid tumor growth and neoangiogenesis, therefore lowering endogenous T4 reduces tumor growth rate. We present the case of two patients with metastatic triple negative breast cancer and metastatic pancreatic cancer respectively, who benefit of the sole treatment with antithyroid drugs and exogenous administration of T3 (liothyronine). In these cases tumor growth was accompanied by T3 depletion in plasma, which may represent a novel marker for progression.
\end{abstract}

Keywords: Neoplasm metastasis, pancreatic neoplasms, thyroid hormones, triiodothyronine, triple negative breast neoplasms

\section{Introduction}

Thyroid function has long been linked to the growth of tumors. Some preclinical studies have attributed T3 a direct negative effect on tumor progression $[8,14$, $15,17]$ as well as chemosensitizing, differentiating and immunomodulatory properties $[1,4]$. Moreover, type 3 deiodinase, which inactivates $\mathrm{T} 3$, has been identified as an oncofetal protein that is expressed in a number of tumors and has been associated with the development, progression and recurrence of cancer $[5,6]$.

On the other hand, evidence has accumulated that L-thyroxine (T4), via a thyroid hormone receptor on plasma membrane integrin $\alpha \mathrm{v} \beta 3$, promotes solid tu-

\footnotetext{
*Corresponding author: Alejandro Rodríguez-Molinero, Fundación Sant Antoni Abat (Consorci Sanitari del Garraf), C/SantJosep 21-23, Vilanovai la Geltru, Spain. Tel.: +34 938931616; E-mail: rodriguez.molinero@gmail.com.
}

mor growth and neoangiogenesis, and there is evidence that lowering $\mathrm{T} 4$ reduces tumor growth rate and enhances radiation and chemotherapy $[2,12]$. Recent experimental evidence suggests that 3,3',5-triiodo-Lthyronine (T3) in physiological concentrations is significantly less (10-100 fold) potent as an inducer of the proliferative action initiated at the plasma membrane integrin $\alpha \mathrm{v} \beta 3$ [13]. Thus, depletion of blood free thyroxine (FT4), along with exogenous T3 supplementation, may therefore be a clinically acceptable and achievable therapeutic goal as a recent experience suggests [10].

In this paper, we present the case of two patients with breast and pancreatic cancers, who were experimentally treated with methimazole induced hypothyroxinemia and exogenous suppressive T3 doses. A temporal stabilization of their neoplasms was observed, and a novel an interesting relation between free-T3 hormone level and the tumor serum markers levels was documented. The latter observation suggests

ISSN 1574-0153/18/\$35.00 (C) 2018 - IOS Press and the authors. All rights reserved

This article is published online with Open Access and distributed under the terms of the Creative Commons Attribution Non-Commercial License (CC BY-NC 4.0). 


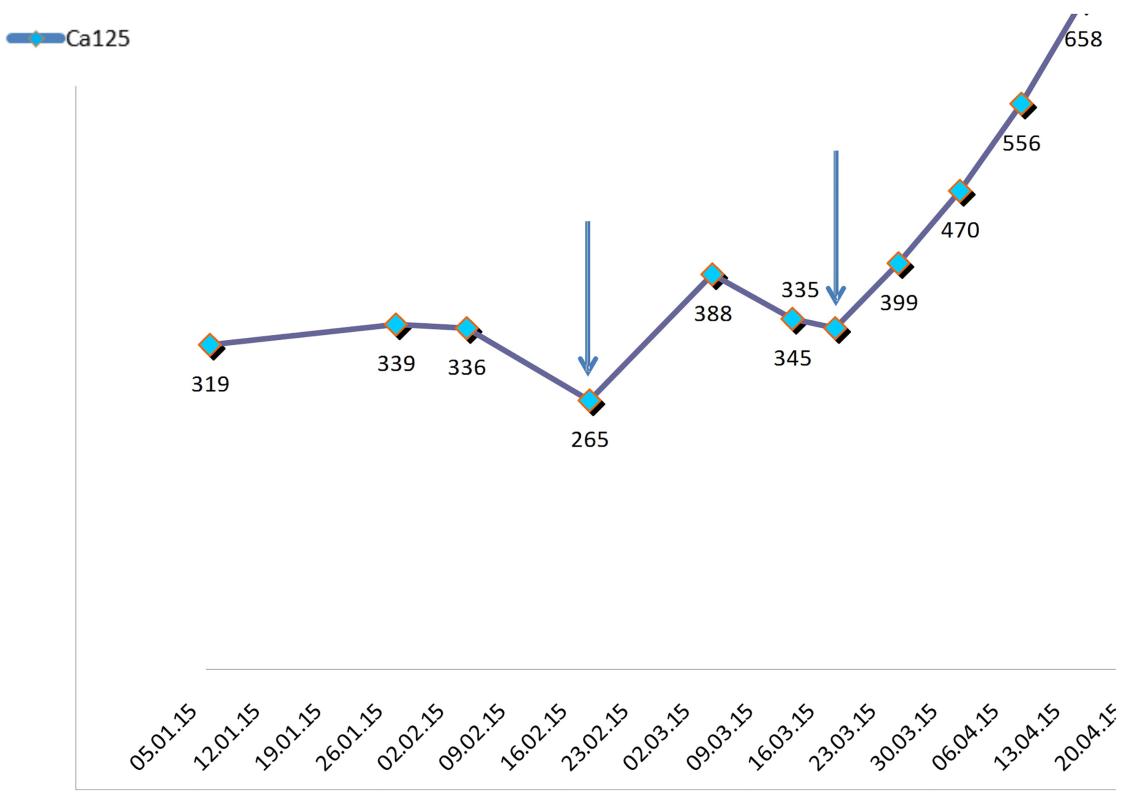

Fig. 1. Initial biochemical responses noted in patient number one, after administration o high doses of liothyronine (L-T3) and methimazolee. Arrows indicate $\mathrm{Ca}-125$ depressions which followed the intermittent treatment administration of liothyronine.

that decreasing free T3 levels may serve as a marker of progression in some cancers.

\section{Case description 1}

A female 67 years old patient with a background of high blood pressure and uterine curettage due to endometrial polyp, presented in September 2012 with an invasive ductal carcinoma of $1.7 \mathrm{~cm}$ maximum diameter in the upper outer quadrant of the right breast (Nottingham Histological Score III/III, negative hormonal receptors ER/PR and HER2, Ki67 50\%) and involvement of 8 out of 10 axillary ganglia. She underwent tumorectomy and three-level axillary lymphadenectomy, being a second lymphadenectomy performed in December 2012 since staging revealed a new lymph node affected in the right armpit. Immediately afterwards, she started docetaxel and carboplatin chemotherapy, with substitution of carboplatin for cyclophosphamide due to hematologic toxicity, and completed the treatment in May 2013. Subsequently, she received radiotherapy on the breast and armpit until August 2013.

In April 2014 she suffered relapse with multiple bilateral lung metastasis (being the larger $0.8 \mathrm{~cm}$ ) detected on a computerized tomography scan (CT) and confirmed by F-FDG TC/PET. She started chemotherapy with paclitaxel (subsequently, nab-paclitaxel because of allergic reaction) plus bevacizumab, which was changed to capecitabine-bevacizumab in November 2014 due to grade III neuropathy. The treatment continued up to March $31^{\text {st }} 2015$, when progression was observed as new left axillary and retropectoral adenopathies.

One month before the mentioned progression (in February 2015) and outside the usual oncologic protocol, the patient accepted to undergo a I-131 scan to investigate whether her neoplasm had functional $\mathrm{Na} / \mathrm{I}$ pump and could be treated with radioactive iodine [16]. Prior to the test, it was confirmed that the patient was euthyroid: TSH $1.29 \mathrm{uUI} / \mathrm{mL}$ (normal range: 0.274.20), total T4 $7.33 \mathrm{ug} / \mathrm{dL}$ (normal range: 5.10-14.10) and total T3 $1.11 \mathrm{ng} / \mathrm{mL}$ (normal range: $0.8-2.0$ ). The preparation for this study involved inhibiting thyroid production with methimazole $15 \mathrm{mg} /$ day and liothyronine $25 \mathrm{mcg} / 8 \mathrm{~h}$ for 3 days. This test ruled out tumor uptake, however, a marked reduction in Ca125 level was observed 12 days later. After repeating the preparation for the test - this time without administering I131 - an equivalent reduction was observed 7 days later (Fig. 1). In a bibliographic review in search of an explanation for this finding, hypothyroxinemia - described by Hercbergs et al. [10] - was found to be a probable cause. The mentioned authors were contacted with the aim of continuing this type of experimental treatment.

The patient was administered methimazole $45 \mathrm{mg}$ per day and increasing doses of liothyronine (L-T3) 


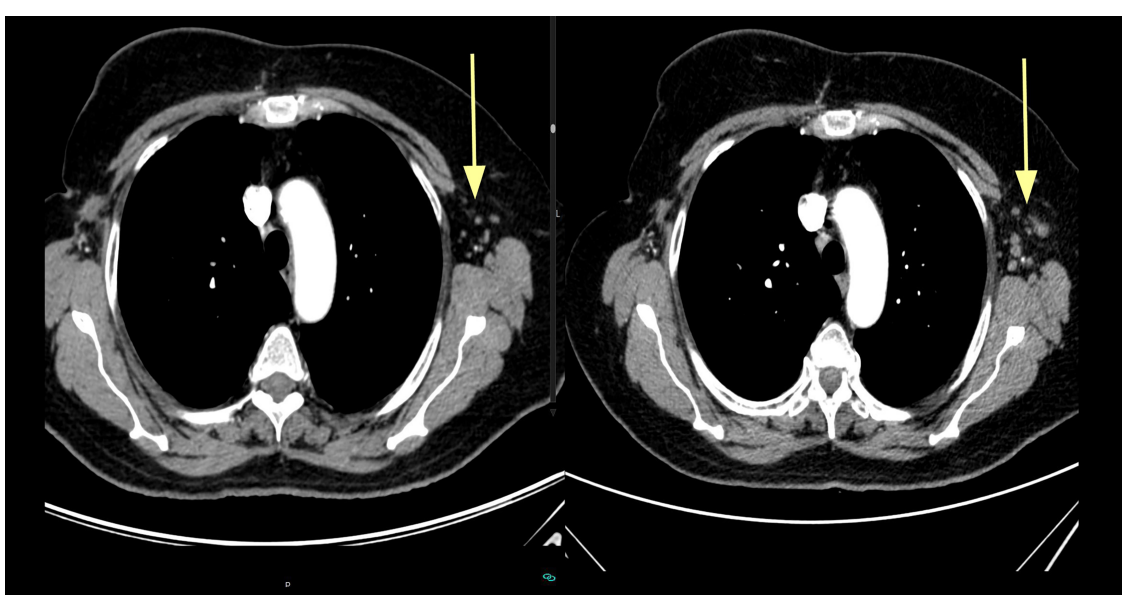

Fig. 2. Tumor progression in axillary lymph nodes.

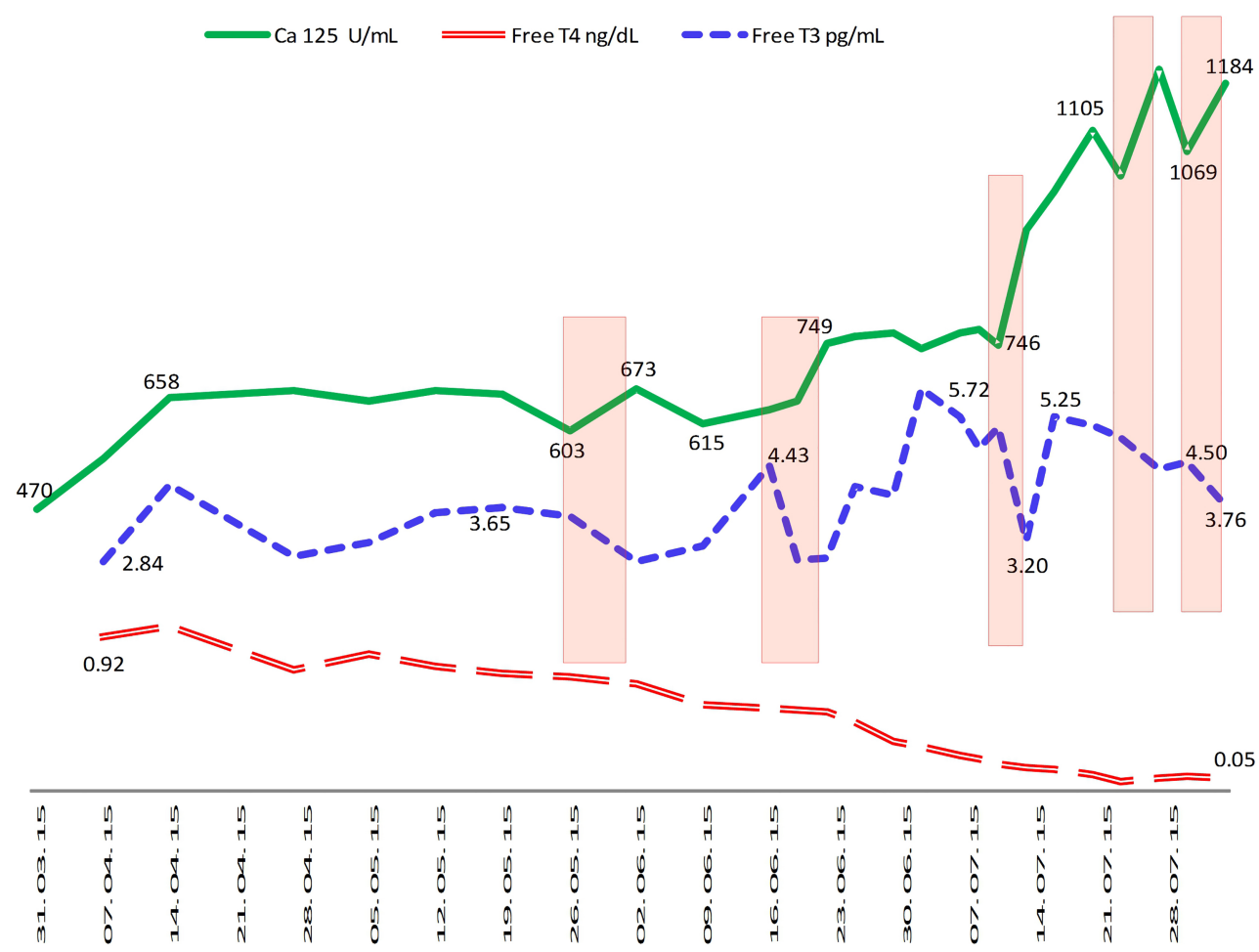

Fig. 3. Evolution of tumor marker (Ca-125), serum free-T3 and serum free-T4 levels in patient number one. It has been consistently shown that a fall in free-T3 lead to an immediate tumor marker rise, irrespective of free-T4 status. Rectangles highlight the graphic sections where this effect can be observed.

until a lower limit TSH was reached (achieved with $6,25 \mathrm{mcg} / 8 \mathrm{~h}$ ). Consequently, the disease was stable for 3 months. In July 2015 increases in Ca 125 were observed, concomitantly with increased left axillary adenopathy found on CT scan (Fig. 2). It was found that each $\mathrm{Ca}-125$ increase was associated with a serum free-T3 fall (Fig. 3) but an increase in oral liothyronine dose and plasma free-T3 level again stabilized Ca-125 levels. Thus, the treatment was continued with progressive liothyronine dose increases (which became progressively insufficient to maintain plasma level of freeT3) until September 2015, when hepatic, mediastinal and pulmonary progression was detected on CT scan. At that point, doxorubicin chemotherapy was added, 


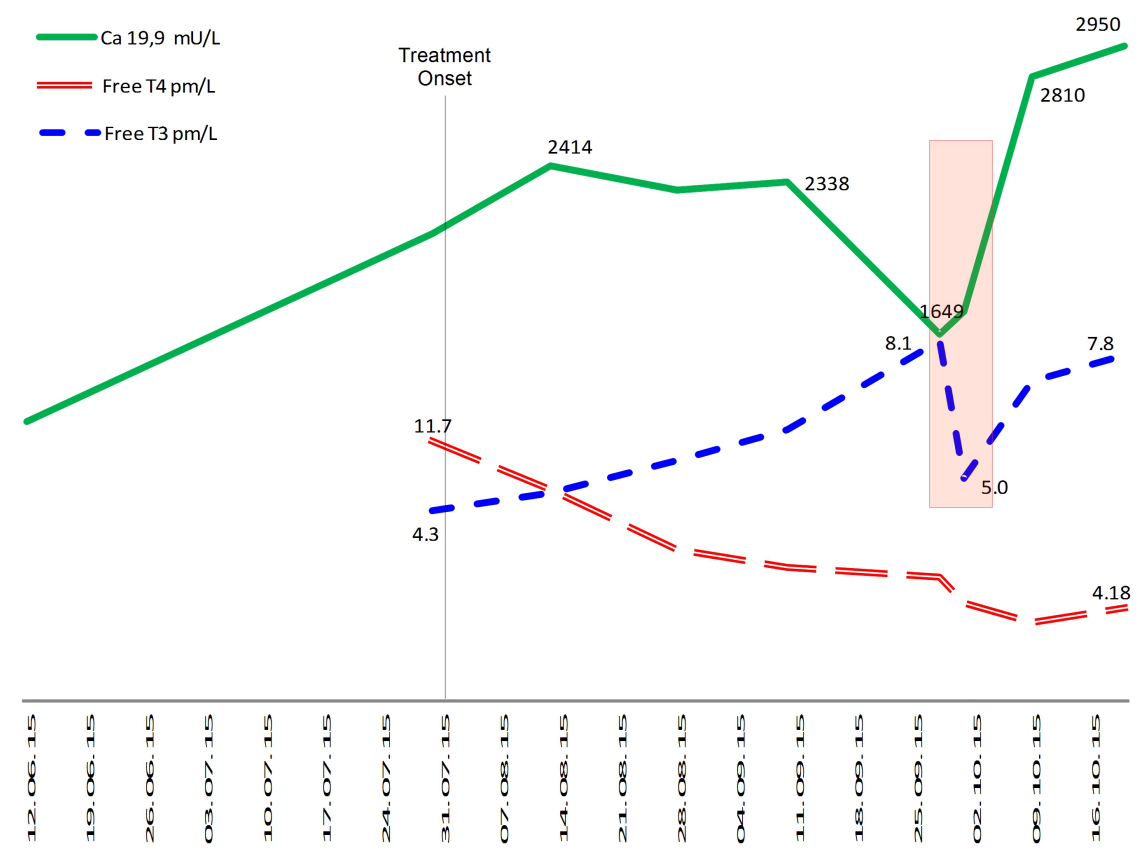

Fig. 4. Evolution of tumour marker (Ca-19.9), serum free-T3 and serum free-T4 levels in patient number two. The rectangle highlights the moment when the tumor marker Ca19.9 rises and serum free-T3 decreases. Serum free-T4 levels, in continuous fall, are apparently unrelated with Ca 19.9 movement.

although it was discontinued on October 2015 (second cycle) because of a reaction to infusion, without any observed tumoral response. On the same date, liothyronine was discontinued (it was $150 \mathrm{mcg} /$ day) because of complex trembling symptoms, which disappeared upon treatment discontinuation. The patient went on with chemotherapy and new experimental drugs until April 2016, when she died probably due to hospitalacquired infection, while staying in hospital with overt hepatic progression. The patient was taking antihypertensive drugs and vitamin D supplementation during the whole course of the disease, while bisoprolol was added during the thyroid treatment to prevent cardiac adverse effects.

\section{Case description 2}

Female 89 years old patient, with past medical history of high blood pressure, depressive syndrome and adenocarcinoma of the anterior surface of the stomach body, treated with subtotal gastrectomy on November 2010 without relapse. In June 2015, she was examined with CT scan because of asthenia and depression. CT findings were compatible with pancreatic cancer with involvement of the splenic vein and lung metastasis. The serum level of Ca19.9 was 1255 UI/Ml (normal
$<37 \mathrm{UI} / \mathrm{mL}$ ). The case was studied by the interdisciplinary commission for tumors, where specific oncologic treatment was dismissed; thus she was offered an experimental treatment with induced hypothyroxinemia [10]. On July $29^{\text {th }} 2015$ she started on methimazole $45 \mathrm{mg} /$ day and increasing doses of liothyronine, distributed in 2-3 daily doses. From the second week on, with $37.5 \mathrm{mcg} /$ day liothyronine dose, Ca $19.9 \mathrm{lev}$ els were observed to stabilize. Eight weeks afterwards, with $43.75 \mathrm{mcg} /$ day liothyronine dose, a marked reduction in $\mathrm{Ca} 19.9$ was observed and a skin metastasis that had appeared during the previous weeks was found to disappear. By that time, methimazole had to be discontinued because of neutropenia. Ten days later, a marked Ca 19.9 increase was observed, concomitantly with a fall in the serum free-T3 levels, while T4 levels remained low (Fig. 4). The treatment with L-T3 was discontinued due to inefficacy on December 2015 and the patient died in January 2016 without undergoing new imaging studies. The patient was taking vitamin D supplementation and betablocking drug bisoprol during the treatment with liothyronine.

\section{Discussion}

In the two cases presented, an inverse relationship was observed between serum free-T3 levels and those 
of the respective tumor markers. In both cases there was a biochemical response to exogenous L-T3, which is not explained by another treatment, since none of the patients received chemotherapy. In addition, in both cases, tumor progression and the increase of serological markers coincided in time with a decline in plasma free-T3, despite the maintenance of or increase of exogenous L-T3 administration.

As T3 was administered exogenously, its plasma clearance can only correspond to an excess of consumption or inactivation of it. This finding could be accounted for by the expression of deiodinase III (DIO3) in tumor cells, which could simultaneously produce a fall in serum free-T3 levels and resistance to treatment. In the first case, this resistance was temporarily overcome by increasing the dose of exogenous L-T3. However it should be noted that the in-vitro data suggests that a 6-fold supranormal concentration of $\mathrm{T} 3$ is necessary to reverse DIO3 expression, thus, while it is plausible that the mechanism of resistance was the deiodinase III, this possibility of requires more rigorous investigation. Since DIO3 expression is difficult to measure in clinical practice, in similar cases, to use blood reverse T3 [rT3] might serve as a non-invasive proxy for tumoral type 3 deiodenase expression.

The consumption of thyroid hormones by tumors has previously been described in the case of hepatic hemangiomas, where it has been confirmed that the phenomena is caused by the expression of tumoral DIO3 [2,9,11]. However, as far as we know, this is the first time that the relationship between plasma clearence of T3 and growth of non-vascular tumors has been documented. In the reported cases, the variations in thyroid hormones were discovered by serendipity since patients were receiving an experimental treatment impacting on thyroid function, however, it is plausible that this may also occur in other euthyroid patients with cancer, in whom the thyroid function is not normally monitored and perhaps should become standard protocol.

It is also important to mention that both patients presented a biochemical tumor response to the treatment, and the regression of a cutaneous metastasis was also observed in the case of the second patient. Hercbergs et al. have previously reported survival advantage in 23 patients under chemotherapy, who underwent treatment with induced hypothyroxinemia and received maintenance doses of liothyronine (TSH in the lower normal limit). In contrast, in our two patients, the tumor response did not seem to be correlated with serum free-T4 levels and was better accounted for by
free-T3 serum changes. The only previous experience we know about cancer treatment with high T3 doses in humans, dates back to 1962, when it was observed that, in combination with steroids, it was beneficial for a group of patients with breast cancer [7], also in 1976 it was successfully used as radiosensitizing substance in newly diagnosed high grade glioma/glioblastoma [18]. In these cases the patients did not receive chemotherapy, but the L-T3 can be chemosensitizing and can also improve the response to the chemotherapy indirectly, due to a secondary decrease of T4 [4]. It is to be investigated in future experiences if combination of $\mathrm{L}$ $\mathrm{T} 3$ and chemotherapy can lengthen the tumor response observed here.

In summary, in the case of our two patients under exogenous L-T3 administration, plasma free-T3 levels were inversely correlated with plasma levels of tumor markers. Exogenous suppressive doses of L-T3 seemed to have direct tumor growth inhibition effect, although it also produced early resistance. Tumor resistance and progression were coincident with increased plasma free-T3 clearance.

\section{Acknowledgments}

In memoriam of Esperanza Molinero Centenera, who with a positive and courageous attitude, contributed to the search for novel treatments to help others with cancer.

\section{References}

[1] V.A. Alamino et al., Antitumor Responses Stimulated by Dendritic Cells Are Improved by Triiodothyronine Binding to the Thyroid Hormone Receptor $\beta$, Cancer Research 75(7) (2015).

[2] J.J. Bergh et al., Integrin alphaVbeta3 contains a cell surface receptor site for thyroid hormone that is linked to activation of mitogen-activated protein kinase and induction of angiogenesis, Endocrinology 146(7) (2005), 2864-2871.

[3] K. Bessho et al., Increased type 3 iodothyronine deiodinase activity in a regrown hepatic hemangioma with consumptive hypothyroidism, European Journal of Pediatrics 169(2) (2010), 215-221.

[4] V. Catalano et al., Activated Thyroid Hormone Promotes Differentiation and Chemotherapeutic Sensitization of Colorectal Cancer Stem Cells by Regulating Wnt and BMP4 Signaling, Cancer Research 76(5) (2016).

[5] D. Ciavardelli, M. Bellomo, C. Crescimanno and V. Vella Type 3 deiodinase: role in cancer growth, stemness, and metabolism, Frontiers in Endocrinology 5 (2014), 215.

[6] M. Dentice and D. Salvatore, Deiodinases: the balance of thyroid hormone: local impact of thyroid hormone inactivation, The Journal of Endocrinology 209(3) (2011), 273-282. 
[7] B. Gardner, A.N. Thomas and G.S. Gordan, Antitumor efficacy of prednisone and sodium liothyronine in advanced breast cancer, Cancer 15(2) (1962), 334-337.

[8] G. Gu et al., Targeting thyroid hormone receptor beta in triplenegative breast cancer, Breast Cancer Research and Treatment 150(3) (2015), 535-545.

[9] A. Güven et al., Severe hypothyroidism caused by hepatic hemangioendothelioma in an infant of a diabetic mother, Hormone Research 63(2) (2005), 86-89.

[10] A. Hercbergs, R.E. Johnson, O. Ashur-Fabian, D.H. Garfield and P.J. Davis, Medically induced euthyroid hypothyroxinemia may extend survival in compassionate need cancer patients: an observational study, The Oncologist 20(1) (2015), 72-76.

[11] S.A. Huang et al., Severe hypothyroidism caused by type 3 iodothyronine deiodinase in infantile hemangiomas, The New England Journal of Medicine 343(3) (2000), 185-89.

[12] H.-Y. Lin, G.V Glinsky, S.A. Mousa and P.J. Davis, Thyroid hormone and anti-apoptosis in tumor cells, Oncotarget 6(17) (2015), 14735-14743.

[13] R. Meng et al., Crosstalk between integrin $\alpha v \beta 3$ and estrogen receptor- $\alpha$ is involved in thyroid hormone-induced proliferation in human lung carcinoma cells, PloS One 6(11) (2011), e27547.
[14] P. Sar, R. Peter, B. Rath, A. Das Mohapatra and S.K. Mishra, 3, 3'5 Triiodo L thyronine induces apoptosis in human breast cancer MCF-7 cells, repressing SMP30 expression through negative thyroid response elements, PloS One 6(6) (2011), e20861.

[15] N. Wang et al., Thyroid hormone inhibits the growth of pancreatic cancer xenograft in nude mice, Nan Fang $\mathrm{Yi}$ Ke Da Xue Xиe Bao = Journal of Southern Medical University 33(8) (2013), 1160-1164.

[16] I.L. Wapnir et al., The Na+/I- Symporter Mediates Iodide Uptake in Breast Cancer Metastases and Can Be Selectively Down-Regulated in the Thyroid, Clinical Cancer Research 10(13) (2004).

[17] C.-C. Yen et al., Mediation of the inhibitory effect of thyroid hormone on proliferation of hepatoma cells by transforming growth factor-beta, Journal of Molecular Endocrinology 36(1) (2006), 9-21.

[18] W.K. Yung, W. Steward, J.E. Marks, M.L. Griem and J.F. Mullan, Glioblastoma multiforme: treatment with radiation and triiodothyronine, International Journal of Radiation Oncology, Biology, Physics 1(7-8) (1976), 645-650. 